\title{
Customised bifurcating networks for mapping polymer dynamics in shear flows
}

Joana Fidalgo, ${ }^{1,3}$ Konstantinos Zografos, ${ }^{1,2}$ Laura Casanellas,,$^{3,4}$ Anke Lindner ${ }^{3}$ and Mónica S. N. Oliveira ${ }^{1}$

${ }^{1}$ James Weir Fluids Laboratory, Department of Mechanical and Aerospace Engineering, University of Strathclyde, G1 1XJ, Glasgow, UK

${ }^{2}$ Present address: School of Engineering, University of Liverpool, Brownlow Hill, L69 3GH, Liverpool, UK

${ }^{3}$ Laboratoire de Physique et Mécanique des Milieux Hétérogènes, CNRS, ESPCI Paris, PSL Research University, University Pierre et Marie Curie, University Paris Diderot, 10 Rue Vauquelin, 75005, Paris, France

${ }^{4}$ Present address: Laboratoire Charles Coulomb, Univ. Montpellier, CNRS UMR 5221, Place Eugene Bataillon-CC069 F34095 Montpellier Cedex 5, France

Understanding the effect of varying shear stresses on individual polymer dynamics is important for applications such as polymer flooding, polymer induced drag reduction or the design of DNA separation devices. In all cases, the individual polymer response to varying shear flows needs to be understood. A biomimetic design rule was recently proposed for bifurcating networks of rectangular channels of constant depth. These customised microfluidic geometries represent an elegant option to investigate, in a single device, multiple well-controlled shear stresses. Here, we present the first experimental realisation of such customised microfluidic networks, consisting of series of rectangular microchannels with varying cross-sections and we demonstrate their potential for testing polymer dynamics. We worked with microfluidic geometries optimised for both Newtonian and power-law fluids and of constant or increasing average wall shear stress. The experimental model systems were tested using particle tracking velocimetry to confirm the theoretically predicted flow fields for shear-thinning xanthan gum solutions and a Newtonian fluid. Then, $\lambda$-DNA molecules were used as an example of a shear sensitive polymer to test the effect of distinct shear stress distributions on their extension. By observing the conformation of individual molecules in consecutive channels, we demonstrate the effect of the varying imposed stresses. The results obtained are in good agreement with previous studies of $\lambda$-DNA extension under shear flow validating the microfluidic bifurcating networks. The customised microfluidic networks can thus be used as platforms for the investigation of individual polymer dynamics, under a large range of well-controlled local and cumulative shear stresses, using a single experiment.

\section{INTRODUCTION}

Microfluidic devices are currently used for numerous types of applications due to cost efficiency, low volume sample consumption and disposability. ${ }^{1,2}$ Some applications are related to cell culture and tissue engineering, ${ }^{3}$ bio-sensing and point-of-care diagnosis, ${ }^{2,4,5}$ particle synthesis ${ }^{6,7}$ or separation. ${ }^{8,9}$ Microfluidics are also used in the context of rheology research since the typical flow conditions achieved at these small scales allow studying the response of solutions and individual particles to imposed stresses in conditions such that viscous and elastic forces dominate relative to inertia. ${ }^{10,11}$ The information gathered from these experiments may help to understand the macroscale behaviour of complex fluids and polymer solutions. ${ }^{12}$ More specifically, bifurcating networks are ubiquitous in these micro-platforms and have been used for varying scopes, ranging from chemistry to biological applications as they offer the ability to mimic certain natural systems (e.g. blood vessels, porous media) and/or carry out many experiments in parallel. Specific examples include the generation of concentration gradients, ${ }^{13,14}$ the study of cell adhesion ${ }^{15,16}$ or $3 \mathrm{D}$ tissue engineering. ${ }^{17,18}$ In the context of polymer dynamics, 
the ability to understand polymer behaviour under varying shear flow conditions is particularly relevant in applications as polymer flooding in porous media for enhanced oil recovery ${ }^{19}$ or the design of DNA manipulation devices ${ }^{20}$. In most cases, the channel dimensions are determined empirically in order to achieve a certain outcome. Experimentally, this translates into an expensive, time-consuming process, where several devices are investigated until optimal results are achieved. Following a design rule when building such devices would make it possible to control local shear stresses and their history and thus to rationalise the design and understanding of such networks.

Here, we present an experimental realisation of a microfluidic bifurcating network following a biomimetic design rule recently proposed by Zografos et $a .^{21}$ which is valid for low inertia flows of both Newtonian and power-law fluids in planar channels of rectangular cross-section. The design rule, which is an extension of Murray's law, ${ }^{22}$ is described by a set of equations that when solved provides the appropriate dimensions of the channels in each subsequent generation of a bifurcating network. A main feature of this design is the ability to control the variation of the average wall shear stress along consecutive network generations, by specifying a branching parameter. Even though the design rule was successfully validated by performing numerical simulations using an in-house computational fluid dynamics (CFD) code ${ }^{23,24}$, an experimental verification of the proposed design rule was still missing.

This study is meant as a proof-of-concept for the use of these customised bifurcating networks to perform shear sensitive tests on complex fluids, using well-controlled conditions. We performed particle tracking velocimetry (PTV) to characterise the flow kinematics inside the channels for geometries of constant and increasing average wall shear stress, using both Newtonian and shear-thinning fluids. After finding a good agreement between experimental (PTV) and theoretical velocity profiles, these geometries were used to probe the response of individual molecules. We chose to use molecules with well-known behaviour under shear flow so these could act as indicators for the good performance of our devices. For this purpose, a dilute solution of lambda bacteriophage DNA molecules ( $\lambda$-DNA) was used as a model to study the customised networks of constant and increasing average wall shear stress. The $\lambda$-DNA molecule was previously studied in several microfluidic devices $^{25,26}$ and it is known to be sensitive to the local shear stress applied. By measuring the degree of deformation of individual $\lambda$-DNA molecules, when flowing along consecutive channels of the network, we were able to demonstrate the predictable effect of the flow conditions imposed by the geometry on the confined flowing sample. We further discuss the experimental limitations inherent to their operation with a view to guide future applications of these devices.

Our study demonstrates the advantage of using bifurcating networks, in which multiple well-controlled shear stresses can be imposed simultaneously, for the investigation of polymer dynamics under a range of shear stresses or shear history in a single device.

\section{MATERIALS AND METHODS}

\section{A. Micro fabrication and network characterisation}

The microfluidic geometries used in this study consist of a series of symmetric bifurcations. Figure 1a presents the schematics of the network where the main channel corresponds to generation $i=0$ and subsequent channels are distributed along generations $i=1,2$ and 3. All networks considered in this work have an 
approximately constant depth $(h)$ throughout the entire domain and the length of each generation $\left(L_{i}\right)$ is set to be twenty times its hydraulic diameter $\left(D h_{i}\right), L_{i}=20 D h_{i}$.

a)

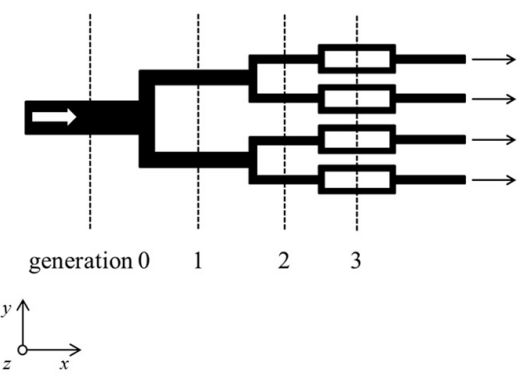

b)

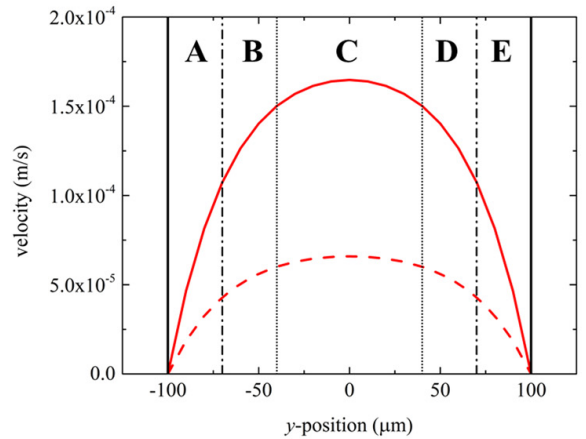

FIG. 1. (a) Schematic representation of a typical bifurcating network used in this study. The four black arrows correspond to the channel outlets from where the fluid is withdrawn. (b) Theoretical parabolic velocity profiles for a Newtonian solution, at flow rates of $40 \mathrm{~nL} \mathrm{~min}{ }^{-1}$ (dashed line) and $100 \mathrm{~nL} \mathrm{~min}{ }^{-1}$ (solid line) for the $z=h / 2$ centreplane of the main channel (generation $i=0$ with width $=200 \mu \mathrm{m}$ and depth $=100 \mu \mathrm{m}$ ); sections A to E used for $\lambda$-DNA molecule analysis are also shown.

The width $\left(w_{i}\right)$ of the channels in each generation $i$ is determined by the biomimetic design rule proposed by Zografos $e t a l^{21}$ according to the specific gradient of the average wall shear stresses desired along the network and the fluid of interest:

$$
\alpha_{i}^{n}\left(1+\alpha_{i}\right)^{n}\left(b_{i}^{*}+\frac{a_{i}^{*}}{n}\right)^{n}=\alpha_{0}^{n}\left(1+\alpha_{0}\right)^{n}\left(b_{0}{ }^{*}+\frac{a_{0}{ }^{*}}{n}\right)^{n}\left(2^{i}\right)^{n} X^{i}
$$

where $\alpha_{0}$ and $\alpha_{i}$ are the aspect ratios (defined as the ratio of depth to width) in the main channel and channels of generation $i$, respectively. The variables $a^{*}$ and $b^{*}$ are geometrical parameters defined as:

$$
\begin{aligned}
a^{*} & =\frac{1}{2\left(1+\frac{1}{\alpha_{i}{ }^{*}}\right)^{2}} \frac{1}{\left[1+4 \sum_{j=0}^{\infty} \frac{(-1)^{j+1}}{\left(\frac{2 j+1}{2} \pi\right)^{3} \cosh \left(\frac{2 j+1}{2} \pi \alpha_{i}^{*}\right)}\right]} \\
a^{*}+b^{*} & =\frac{1}{2\left(1+\frac{1}{\alpha_{i}^{*}}\right)^{2}} \frac{3}{\left[1-\frac{1921}{\pi^{5} \alpha_{i}^{*}} \sum_{j=1,3,5, \ldots j^{5}}^{\infty} \tanh \left(\frac{j \pi \alpha_{i}^{*}}{2}\right)\right]}
\end{aligned}
$$

and $X$ is the branching parameter, which relates the average wall shear stress in the channels of different generations $\left(\overline{\tau_{i}}\right)$ with the average wall shear stress imposed in the main channel $\left(\overline{\tau_{0}}\right)$ :

$$
X^{i}=\frac{\overline{\tau_{i}}}{\overline{\tau_{0}}}
$$

Please note that in equations 2 and 3 , the aspect ratio $\alpha_{i}{ }^{*}$ is defined as

$$
\alpha_{i}^{*}=\left\{\begin{array}{l}
\frac{h}{w_{i}} \text { if } h \leq w_{i} \\
\frac{w_{i}}{h} \text { if } h>w_{i}
\end{array}\right.
$$


TABLE I. Channel dimensions.

\begin{tabular}{cccccc}
\hline Geometry & Dimension $(\mu \mathrm{m})$ & \multicolumn{4}{c}{ Channel generation $(i)$} \\
& & 0 & 1 & 2 & 3 \\
\hline \multirow{2}{*}{$X=1$} & $w$ & 202.5 & 120.0 & 80.0 & 55.0 \\
& $h$ & 97.8 & 97.5 & 97.0 & 96.5 \\
\hline \multirow{2}{*}{$X=1.9$} & $w$ & 200.0 & 80.0 & 40.0 & 26.0 \\
& $h$ & 100.9 & 100.5 & 100.5 & 100.0 \\
\hline
\end{tabular}

The design rule is suitable for both Newtonian and power-law fluids, exhibiting a shear-dependent viscosity:

$$
\eta=k \dot{\gamma}^{n-1}
$$

where $\eta$ is the viscosity, $k$ the consistency index, $\dot{\gamma}$ the magnitude of shear rate and $n$ the power-law index of the fluid (for $n=1$, the viscosity is not shear dependent and the Newtonian model is recovered). In order to find the solution, $\alpha_{i}=h / w_{i}$, the bisection method is employed similarly to the previous works of Zografos et al $l^{11}$ for power-law fluids and of Emerson et al $^{27}$ for Newtonian fluids $(n=1)$.

Geometries are named according to the gradient of average wall shear stress imposed along the network using the branching parameter $X$. In this work, we consider two geometries: $X=1$ corresponding to a network with uniform average wall shear stress and $X=1.9$ corresponding to a network with increasing average wall shear stress along the consecutive generations. A CAD drawing of the channel geometries was first prepared and printed in a film photomask. The epoxy mould (SU8-100, Microchem) with the same design was fabricated by photolithography and the corresponding channel widths and depths were measured by optical microscopy and surface profilometry (Dektak 6M, Veeco), respectively.

The dimensions for the two geometries used are given in Table 1. The small differences in depth between channels in the same network is inherent to the fabrication technique used and were taken into consideration to ensure that all visualisation experiments were performed at the channels' centreplane (i.e. for $z=h / 2$ ).

The microchannels used in the experiments were produced in PDMS (polydimethylsiloxane, Sylgard 184, Dow Corning) by soft lithography and bonded to a glass slide using oxygen plasma (Zepto, Diener).

\section{B. $\lambda$-DNA solution and rheological characterisation}

As an example of a shear sensitive molecule, $\lambda$-phage DNA (Sigma-Aldrich, $48 \mathrm{kbp}, \mathrm{Mw}=31.5 \times 10^{3} \mathrm{kDa}$ ) was used in this study. The corresponding contour and persistence lengths are $16.3 \mu \mathrm{m}$ and $50 \mathrm{~nm}$, respectively. ${ }^{28}$

The experiments were performed using a slightly viscous buffer solution composed by $10 \mathrm{mM}$ Tris buffer $(\mathrm{pH}=8.0), 10 \mathrm{mM} \mathrm{NaCl}, 1 \mathrm{mM}$ EDTA, $\beta$-mercaptoethanol $4 \%(\mathrm{v} / \mathrm{v})$, oxygen scavenger solution and sucrose. Both $\beta$-mercaptoethanol and the oxygen scavenging solution (the former composed of glucose, glucose oxidase and catalase) were added to the main solution in order to remove the reactive species of $\mathrm{O}_{2}$, which are responsible for photo bleaching.

$\lambda$-DNA was fluorescently labelled using YOYO-1 dye (YOYO-1 Iodide (491/509) - $1 \mathrm{mM}$ solution in DMSO, Life Technologies) using the procedure described in Teclemariam 200729. $29.2 \mu \mathrm{L}$ of a $\lambda$-DNA intermediate solution $(2 \mu \mathrm{g} / \mathrm{mL})$ and $3.6 \mu \mathrm{L}$ of YOYO-1 intermediate solution $(5 \mu \mathrm{M})$ were added to the final 
sample such that the homodimer would bind to the $\lambda$-DNA double strand at a 1:5 dye: bp ratio. The labelling process takes place overnight, at room temperature, under gentle stirring and the resultant contour and persistence lengths of the labelled molecule increase to $21-22 \mu \mathrm{m}$ and $70 \mathrm{~nm}^{29}$, respectively.

The steps of polymer dilution in the buffer solution and subsequent fluorescent labelling with YOYO-1 were performed in the dark to avoid photo bleaching. More details on sample preparation can be found in the supplementary material, Section S1.

The concentration of $\lambda$-DNA in the solutions used in the experiments is $0.04 \mathrm{ppm}$. This value is significantly lower than the overlap concentration $c^{*}$ of 36.4 ppm estimated as $^{30}$

$$
c^{*}=3 M_{w} /\left(N_{A} 4 \pi R_{g}{ }^{3}\right)
$$

where $M_{w}$ is the $\lambda$-DNA molecular weight and $N_{A}$ the Avogadro's number. For such dilute solutions, molecules are expected not to interact with each other in the surrounding medium, allowing their individual characterisation in a simple shear flow.

The $\lambda$-DNA in viscous buffer solution was characterised using a rotational rheometer (Physica MCR 501, Anton Paar) with a cone-plate geometry $\left(60 \mathrm{~mm}, \theta=1^{\circ}\right)$ in the range $0.1<\dot{\delta}<1000 \mathrm{~s}^{-1}$, at a temperature of $20{ }^{\circ} \mathrm{C}$ (which corresponds to the temperature set for the microfluidic experiments). As expected, the solution exhibited a constant shear viscosity $\left(38.2 \times 10^{-3} \pm 0.015 \mathrm{~Pa} \mathrm{~s}\right)$ as a result of the low polymer concentration.

\section{Experimental setup and data acquisition}

\section{Particle Tracking Velocimetry (PTV)}

PTV experiments were performed with a Newtonian fluid without $\lambda$-DNA to confirm the velocity fields in the regions where visualisation of $\lambda$-DNA transport dynamics will be carried out on both $X=1$ and $X=1.9$ geometries. For that purpose, fluorescent tracer particles were added to distilled water in small concentrations so that individual particles could be tracked along consecutive frames without being mistaken with other surrounding particles. The particles are $1 \mu \mathrm{m}$ in diameter and have excitation and emission wavelengths of 535 $\mathrm{nm}$ and $575 \mathrm{~nm}$, respectively. The experiments were performed on an inverted microscope (AX 10, Zeiss) employing a 63x water immersed magnification objective with numerical aperture NA = 1.2 (C-Apochromat, Zeiss) and a HXP-120 Light Source for fluorescence illumination. The images were acquired using a high-speed camera (FASTCAM SA4, Photron) and both the time between frames and the exposure time were adjusted according to the flow conditions, so that the particles could be successfully tracked. The images were analysed using Image $\mathrm{J}$ and Matlab to determine the average velocity of each detected particle.

The fluid was withdrawn from the 4 outlets of the geometry, using a precision syringe pump (Nemesys, Cetoni) and four $100 \mu \mathrm{L}$ glass syringes. This protocol ensured identical flow rates in all channels of each generation.

The experimental velocity profiles obtained by PTV were compared with the analytical profiles for fullydeveloped flow of a Newtonian fluid in a channel of rectangular cross-section: ${ }^{31}$

$$
u_{x}(y, z)=\frac{48 Q}{\pi^{3} h w} \frac{\sum_{n, o d d}^{\infty} \frac{1}{n^{3}}\left[1-\frac{\cosh \left(n \pi \frac{y}{h}\right)}{\cosh \left(n \pi \frac{w}{2 h}\right)}\right] \sin \left(n \pi \frac{z}{h}\right)}{\left[1-\sum_{n, o d d}^{\infty} \frac{192 h}{n^{5} \pi^{5} w} \tanh \left(n \pi \frac{w}{2 h}\right)\right]}
$$


where $u_{x}$ is the $x$-component of the velocity, $Q$ is the imposed flow rate, $w$ and $h$ are the width and height of the channel, respectively, with $0 \leq z \leq h$ and $-w / 2 \leq y \leq w / 2$. In this case, the $z$-position for which the measurements were taken corresponds to half of the channel height $(z=h / 2)$.

The shear rate profiles are determined using equation 9, which has been simplified as the experiments were performed at the channel mid height where the corresponding velocity derivative relative to $z$ is zero.

$$
\dot{\gamma}=\sqrt{\left[\frac{-\Delta P}{2 \mu L} \sum_{n=0}^{\infty} a_{n} \cos \left(\frac{2 \lambda_{n} z}{H}\right) \frac{2 \lambda_{n}}{H} \sinh \left(\frac{2 \lambda_{n} y}{H}\right)\right]^{2}}
$$

where $\lambda_{n}=\frac{(2 n+1) \pi}{2}, a_{n}=\frac{H^{2}(-1)^{n}}{\lambda_{n}{ }^{3} \cosh \left(\lambda_{n} \frac{W}{H}\right)}, H$ is the channel height and $W$ is the channel width. We calculated the average shear rate in each branch for the channel sections considered in Figure $1 \mathrm{~b}$.

\section{A-DNA visualisation}

$\lambda$-DNA visualisation experiments were performed using the same pumping protocol, microscope and illumination source, but a CCD camera (Orca Flash 4.0, HAMAMATSU) was used to determine the $\lambda$-DNA molecule extension. All the videos were recorded at the channels centreplane $(z=h / 2)$. A dichroic filter was selected to adjust the appropriate wavelengths for the excitation and emission of $\lambda$-DNA-YOYO-1 complex (491 $\mathrm{nm}$ and $509 \mathrm{~nm}$, respectively). ${ }^{32}$

$\lambda$-DNA was fluorescently stained and the exposure time was set to $\sim 60 \mathrm{~ms}$ in order to observe its structure with satisfactory contrast and definition. As a consequence, molecules traveling at large velocities appeared as blurred elongated bodies, leading to erroneous measurements biased towards larger extensions than reality. To mitigate this problem, the microscope stage was displaced in the opposite direction of the flow at constant speed. Several distinct velocities were used according to the flow rate and channel under consideration in order to follow molecules positioned across the whole channel width during image acquisition. This allowed us to obtain sharp images and to capture precisely the typical deformation behaviour of a single molecule, such as stretching, recoiling and tumbling along the channel length. ${ }^{33}$ It is important to note that for the procedure used for the visualisation, each molecule could be tracked for long enough to cover the residence time in each channel before significant photo bleaching occurs.

The molecules were considered deformed if their maximum extension was larger than $1.5 \mu \mathrm{m}$, which is approximately two times the considered radius of gyration for the labelled molecule using TOTO- 1 dye $(0.73$ $\mu \mathrm{m}) .{ }^{34}$ This parameter corresponds to the radius of the coil formed by the polymer complex in its relaxed state since $\lambda$-DNA is a semi-flexible polymer. So, for sizes smaller than $1.5 \mu \mathrm{m}$, it was assumed that the molecule was in equilibrium, this is, no deformation is considered. The microscopic depth of field (dof) was estimated to be $2.07 \mu \mathrm{m} .^{35}$ 
In order to simplify the analysis, the channel width was divided into different areas (Figure 1b) and an average of molecule extension $\langle x\rangle$ was determined for each of these areas. More specifically, for $i=0$ and $i=1$, five sections were considered: $\mathrm{A}$ and $\mathrm{E}$ correspond to the areas close to the channel lateral walls (each one corresponding to $15 \%$ of the total channel width); B and D correspond to intermediate sections of the channel ( $15 \%$ of the total channel width) and a large section $\mathrm{C}$, in the centre of the channel, occupying $40 \%$ of the total width. For $i=2$ and $i=3$, it is more difficult to accurately define the molecule position in the lateral sections, given the small width of the channels. For these cases, it was decided to consider an identical area in the middle (section C, $40 \%$ of total channel width) and the two lateral areas close to the channel walls grouped together (A $+\mathrm{B}$ and $\mathrm{D}+\mathrm{E}$, each occupying $30 \%$ of the total channel width).

\section{RESULTS AND DISCUSSION}

In this section, we validate the proposed design of the networks and demonstrate their potential to examine the behaviour of bio sensitive molecules. We have chosen $\lambda$-DNA as this is a well characterised molecule in terms of behaviour under simple shear. ${ }^{36}$ First, we analyse the velocity field in the various generations to ensure bulk fluid flow kinematics are as expected; secondly, we examine the individual dynamics of $\lambda$-DNA along a channel, and we then make full use of the customised geometries to study the effect of shear rate on the molecules' maximum extension.

\section{A. Velocity profiles characterisation}

The velocity profiles obtained by PTV, for the various branches of geometries $X=1$ and $X=1.9$, are presented in this section. To simplify the PTV analysis, a Newtonian fluid without the presence of $\lambda$-DNA is used to quantify the velocity profiles in the regions of interest, where the flow is expected to be fully-developed. The normalised axial velocity profiles along $y$ at the $z=h / 2$ centreplane (scaled here using the average inlet velocity $\overline{u_{0}}$ ) for each generation of the network (cf. Figure 2a for $X=1$ and Figure $2 \mathrm{~b}$ for $X=1.9$ ) exhibit a parabolic shape, which is in good agreement with the theoretical profile evaluated by equation 8 . Figures $2 \mathrm{c}$ and $2 \mathrm{~d}$

illustrate the evaluated normalised shear rates (scaled here with the average shear rate at the inlet channel $\overline{\dot{\gamma}_{0}}$, being $\overline{\dot{\gamma}_{0}}=2 \overline{u_{0}} / 0.5 w_{0}$ ) in the observation areas considered for each generation.

For both geometries it can be seen that the shear rate in the central section (Section $\mathrm{C}$ ) is smaller, and increases for the sections closer to the walls (Sections $\mathrm{A}, \mathrm{B}, \mathrm{D}$ and $\mathrm{E}$ ). For $X=1$, the maximum velocity decreases along the network as the flow divides (Figure 2a) while for $X=1.9$, the maximum velocity variation along the generations is non-monotonic according to the channel width and flow rate (Figure $2 b$ ). As a result, the average shear rates in the various sections exhibit similar values for all generations in geometry $X=1$ and can be considered nearly constant (Figure 2c), while a strong increase along consecutive generations is observed for $X=1.9$ (Figure 2d) as expected by setting a design with branching parameter greater than unity $(X>1$ predicts increasing average wall shear stresses). For these points, the horizontal error bars correspond to the channel width of the corresponding section and the vertical error bars indicate the range of shear rates found in that same section. Then in each subsequent generation a different range of average shear rates is achieved and one can impose a large range of flow conditions in a single experiment by considering all the channels along the consecutive network generations. It is also worth to note that due to Poiseuille flow profile in each channel, we 
can also obtain several average shear rates for a given generation of the network, corresponding to the different regions considered in each channel (Figure 1b).
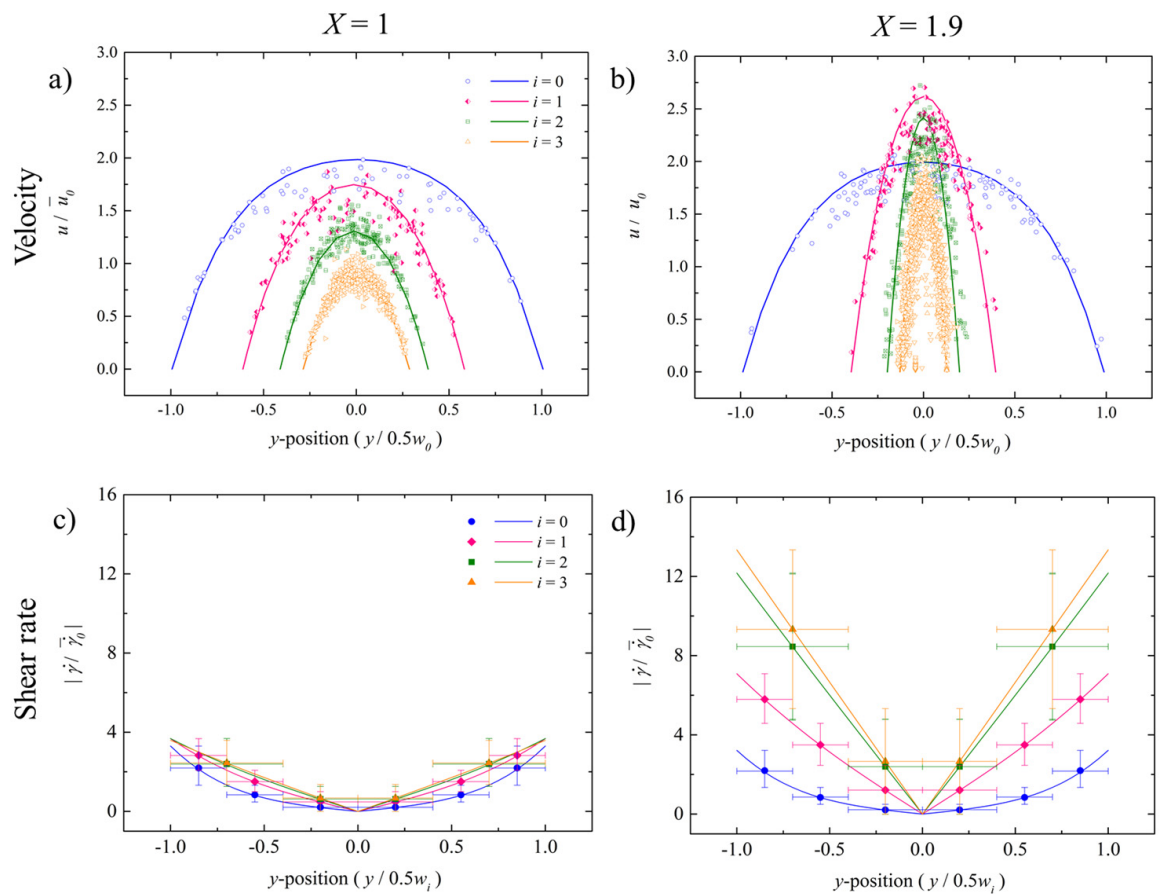

FIG. 2. Flow kinematics in the various generations of geometries $X=1(\mathrm{a}, \mathrm{c})$ and $X=1.9(\mathrm{~b}, \mathrm{~d})$. Comparison between the normalised velocity profiles (local velocity $u$ is normalised using the average velocity in the main channel, $\overline{u_{0}}$ ) obtained by experimental PTV (symbols) and theoretical prediction (solid lines) for Newtonian fluids in geometry $X=1$ (a) and $X=1.9$ (b) at $z=h / 2$ centreplane. Corresponding normalised shear rate profiles (using the average shear rate in the main channel, $\overline{\dot{\gamma}_{0}}=2 \overline{u_{0}} / 0.5 w_{0}$, as reference) for network $X=1$ (c) and $X=1.9$ (d); the symbols represent the average value in each of the sections used for the analysis (cf. Figure 1b). Note that the $y$-coordinate has been shifted so that $y=0$ corresponds to the centreline of each channel.

As observed in Figure 2d, the increase in shear rate from generations $i=2$ to $i=3$ (geometry $X=1.9$ ) is smaller when compared to the increment between the first generations (from $i=0$ to $i=1$ and $i=1$ to $i=2$ ). When producing the mould from a film mask using photolithography, there are typically small differences (within $\pm 5 \mu \mathrm{m}$ ) between the theoretical and real channel widths due to errors inherent to the manufacturing process. In general, these deviations effectively impose a limit in the smallest dimension of channels that can be used to achieve a required accuracy in terms of the average wall shear stress. In our particular case, manufacturing errors result in non-negligible relative deviations for the smallest channels (i.e. the last generation) of geometry $X=1.9$ thus limiting the number of generations that can be used accurately in experimental model systems. A more detailed analysis of the impact of manufacturing errors, and associated discussion, is provided in supplementary material, Section S2.

Taking advantage of the universality of the $X=1$ geometry (i.e the same design is valid for both Newtonian and a range of power-law fluids ${ }^{21}$ ), the same chip was used to test two different xanthan gum solutions, which exhibit a shear thinning behaviour $(n=0.62$ and $n=0.79)$. These results are reported in supplementary material (Section S3). The velocity profiles are flattened compared to the Newtonian case as expected for shear thinning fluids and are in good agreement with the numerical simulations. 
Summarising the previous findings, an overall good agreement was found between the experimental velocity profiles determined by PTV and both theoretical and numerical velocity profiles obtained for Newtonian and power-law fluids, respectively. This provides confidence in the experimental biomimetic geometries for further use with specific applications.

\section{B. Behaviour of shear sensitive biopolymers in customised networks}

\section{Dynamics of $\lambda$-DNA molecules}

Individual molecule deformation in a shear flow is known to exhibit a combination of different mechanisms such as tumbling, stretching and recoiling, depending on the polymer relaxation time and the shear rate. ${ }^{36}$

Figure 3a presents an example of a typical deformation sequence experienced by a $\lambda$-DNA molecule in a Newtonian medium transported along the last generation $(i=3)$ of geometry $X=1.9$. These images were obtained by displacing the microscope stage at appropriate velocities as explained in the Experimental section (Section II.C.2), which allowed us not only to record the full deformation cycle of each examined molecule, but above all to capture sharp images in which the length observed and measured is solely due to the molecule extension rather than a technical artefact associated with the large exposure times used.

a)

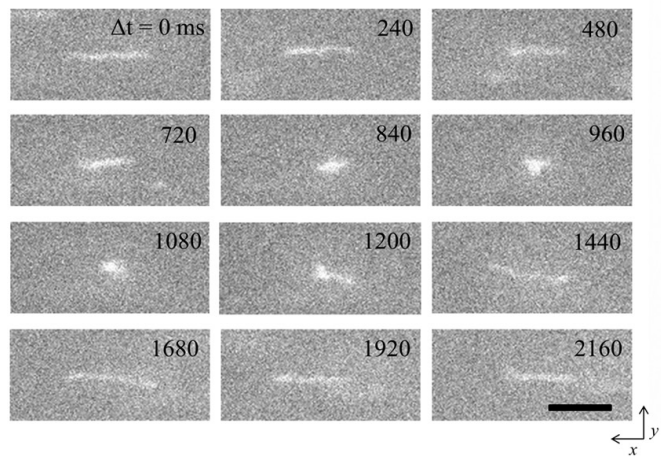

b)

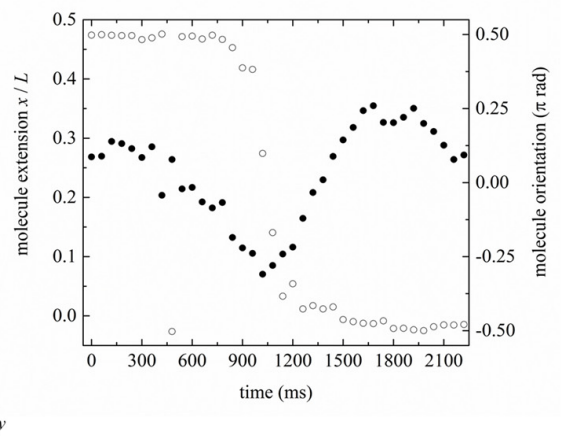

FIG. 3. (a) Typical images of $\lambda$-DNA molecule undergoing deformation in a Newtonian viscous solution;

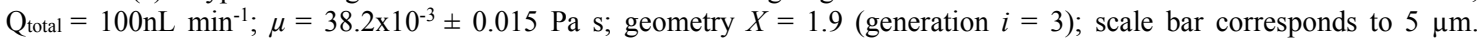
(b) Evolution of molecule extension $(\bullet)$ and orientation $(\circ)$ corresponding to the deformation cycle presented in Figure $3 \mathrm{a}$.

The corresponding molecule extension and molecule orientation are presented in Figure $3 \mathrm{~b}$. The molecule is fitted by positioning an ellipse around it. The extension is quantified as the absolute fractional extension $(x / L)$, with $x$ being the instantaneous molecule length, given by the major axis of the ellipse, measured for consecutive frames and $L$ the reference value corresponding to the contour length of fluorescently labelled molecules (21 $\mu \mathrm{m}$ ). Molecule orientation is given by the angle between the major axis of the ellipse and the $x$-axis (streamwise direction).

The molecule shown in Figure $3 \mathrm{a}$ is positioned close to the channel wall ( $4-5 \mu \mathrm{m}$ away from the wall), where the shear rate is estimated to be $\sim 15 \mathrm{~s}^{-1}$ and thus the $\lambda$-DNA molecule is subjected to a high shear stress. For a certain period of time, the molecule moves in an extended configuration, parallel to the $x$-axis $(\theta \sim+\pi / 2$ radians). At $\Delta t=0 \mathrm{~ms}$, the molecule starts to retract and the angle relative to the $x$-axis decreases slightly, corresponding to the rotation experienced by the molecule. This is a smooth process until the molecule recovers 
the coiled configuration at $\Delta t \sim 1020 \mathrm{~ms}\left((x / L)_{\text {minimum }} \sim 0.07\right)$; meanwhile, the molecule rotates and starts stretching again until a maximum extension is reached for $\Delta t \sim 1680 \mathrm{~ms}(x / L \sim 0.35 ; \theta \sim-\pi / 2$ radians $)$. At this point, the extended molecule is again aligned with the $x$-axis $(\theta \sim-\pi / 2$ radians or $+\pi / 2$ radians $)$ and the previously described cycle may begin again.

\section{A-DNA molecule extension along the networks}

In this section, we evaluate the maximum extension observed for each analysed $\lambda$-DNA molecule during its cycle of deformation to quantify the average value in the various sections of each generation in the two networks $(X=1$ and $X=1.9)$. We analysed an average of 50 molecules in each channel section to guarantee good statistics.

Figure 4 presents the mean fractional extension $\langle x>/ L$ (where $\langle x>$ is now the average value of the maximum extension measured for the molecules within the observation regions defined in Figure $1 \mathrm{~b}$ ) for a total flow rate of $40 \mathrm{~nL} \mathrm{~min}{ }^{-1}$. For the geometry with $X=1$, the molecule deformation observed is modest (Figure 4a) although higher at the walls than at the centre as expected given the shear rate distribution (Figure 2c). The mean fractional extension value of $\sim 0.1$ is a result of the molecule fluctuations, commonly observed due to Brownian motion. ${ }^{36}$ The values of mean fractional extension for the molecules traveling along the channels of all generations practically superimpose. On the contrary, for the $X=1.9$ geometry (Figure $4 \mathrm{~b}$ ), the average wall shear stress is expected to increase along the consecutive generations and we observe a significant increase on molecule average extension along the various generations in the network. The higher shear stress at the walls in subsequent generations leads to a stronger hydrodynamic drag force acting on the molecule. If this force overcomes the entropic forces responsible for keeping the $\lambda$-DNA in a coiled structure, then the molecule will stretch and the average elongation is expected to increase in the range of shear stresses applied until a plateau of $0.4-0.5$ is reached in shear flows. ${ }^{36,33}$ As a consequence of the distinct shear rate profiles generated in the geometry $X=1.9$ (Figure $2 \mathrm{~d}$ ), the values for $\lambda$-DNA extension could be obtained for a large set of shear rate conditions, using a single imposed flow rate.
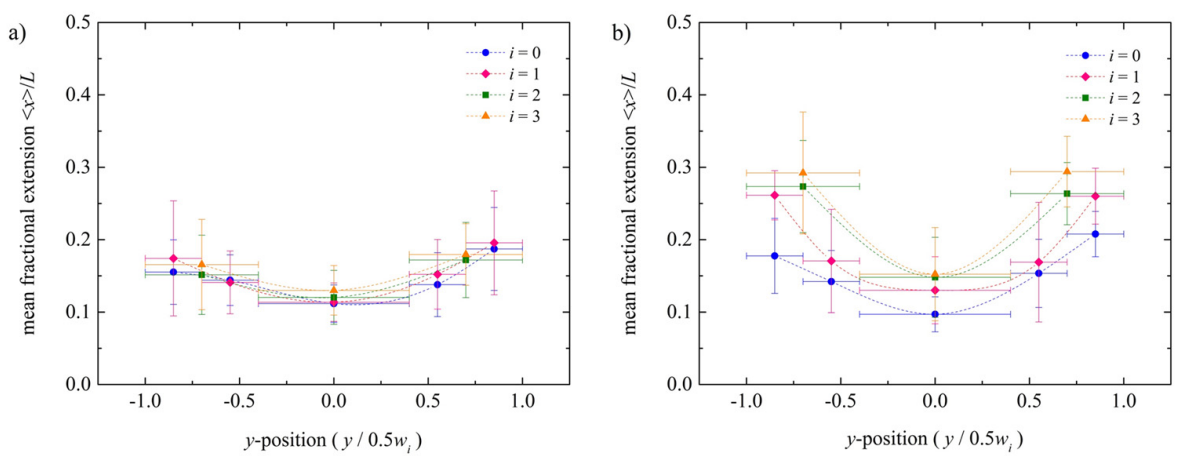

FIG. 4. Mean fractional extension of $\lambda$-DNA molecules determined for each section of the channels (as defined in Figure 1b) along the various generations of the geometries $X=1$ (a) and $X=1.9$ (b) at $Q_{\mathrm{t}}=40 \mathrm{~nL} \mathrm{~min}{ }^{-1}$. Dashed lines are guidelines connecting the average molecule extension in the different channel sections. 
The error bars in Figure 4 represent the standard deviation of the molecule elongation for each specific section of the channel. These relatively large values of the standard deviation may be a combined result of the range of shear stresses the molecules are subjected to in each channel section under analysis, and the so called "molecular individualism" of $\lambda$-DNA, describing the unpredictability of individual molecule behaviour under similar flow conditions. ${ }^{37}$ Simple shear flow is characterised by the contribution of both fluid elongation and vorticity in the same proportion. As such, the deformation of the $\lambda$-DNA semi-flexible molecule is a combination of different behaviours, such as stretching, tumbling and recoiling. Typically, after molecule stretching and aligning along the $x$-axis, the hydrodynamic forces decrease and the molecule retracts until it reaches an equilibrium shape, i.e. the coiled shape. Because of the molecule size and Brownian motion, the molecule feels the rotational component of the flow, resulting in tumbling phenomena. ${ }^{38}$ Since this deformation is stochastic, the maximum length registered can change for the set of molecules analysed in the same channel section. ${ }^{37}$

To corroborate the good performance of the microfluidic designs, we now concentrate on the region close to the wall for which the devices tested are expected to give us the ability to increase $(X=1.9)$ or maintain $(X=1)$ the average shear stresses at the wall along subsequent generations. Figure 5 presents the comparison between the maximum fractional lengths in the lateral sections of the channels (i.e. in the sections close to the walls as shown in Figure 1b), along the four generations in both geometries at the flow rate of $40 \mathrm{nLmin}^{-1}$. It is clear that for geometry $X=1$, the maximum extension is similar for all generations, while for $X=1.9$, the maximum extension becomes stronger when progressing along the geometry in accordance to the wall shear stress variation along the network as prescribed by the branching parameter selected.

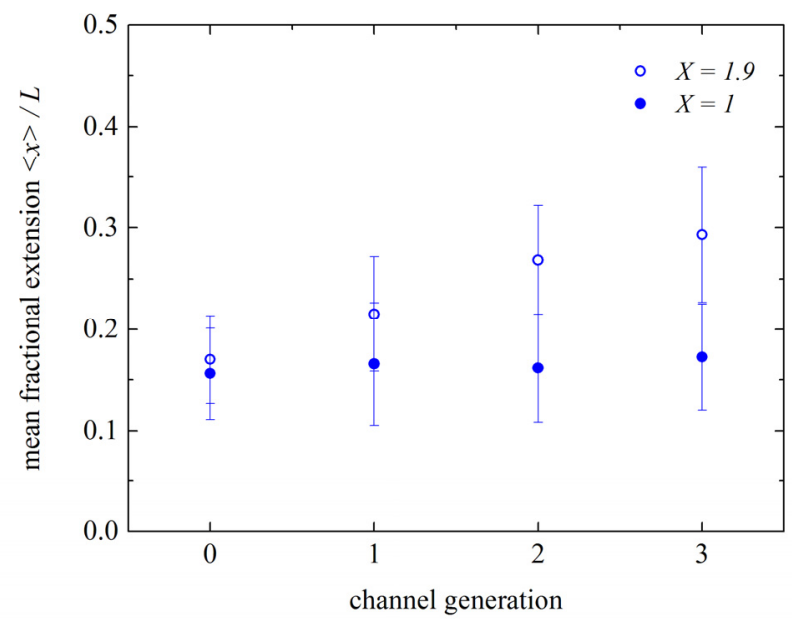

FIG. 5. Comparison between mean fractional extension of the molecules in the lateral channel sections for both

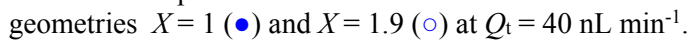

To summarise, the data set collected in all our experiments is plotted in Figure 6 as a function of Weissenberg number (Wi), defined as the product of the longest relaxation time of the polymer $\left(\mathrm{t}_{\text {relaxation }}\right)$ and the shear rate $(\dot{\gamma})$. Here, to evaluate the shear rate, we considered the average value $\left(\overline{\dot{\gamma}_{i}}\right)$ for each channel section. The relaxation time was estimated to be $3.6 \mathrm{~s}$ based on the relaxation time determined for a similar 
molecule complex in a sucrose buffer solution of $41 \mathrm{cP}$ viscosity. ${ }^{39}$ The evolution of the mean fractional extension with the Wi number is in good agreement with the trends observed by Smith et al. ${ }^{36}$ and Teixeira et $a l .{ }^{33}$ for the extension of $\lambda$-DNA in simple shear flow using two parallel plates. In their work, $\lambda$-DNA molecules were also labelled using YOYO-1 dye at a similar proportion as used here (1:4 dye: bp ratio).

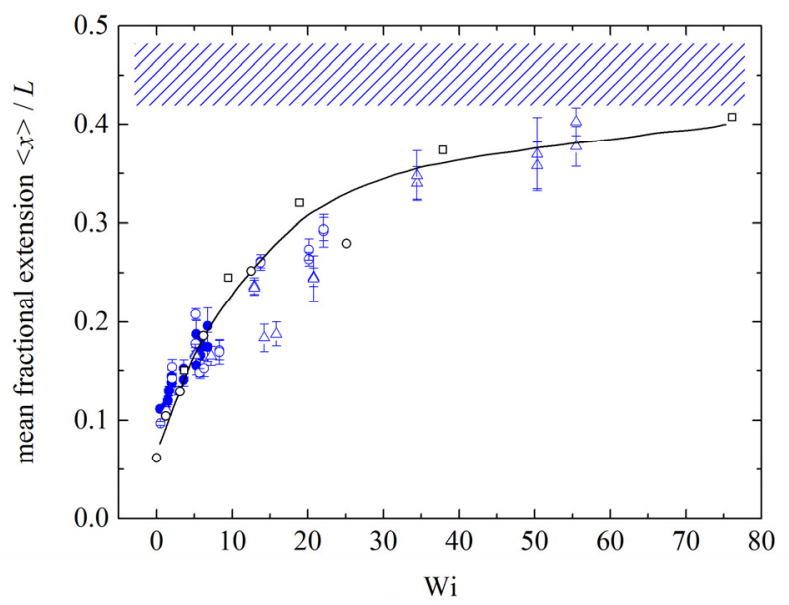

FIG. 6. Mean fractional extension of $\lambda$-DNA as a function of Wi number for geometries $X=1$ for $\mathrm{Q}_{\mathrm{t}}=40 \mathrm{nLmin}^{-1}(\bullet), X=$ 1.9 for $\mathrm{Q}_{\mathrm{t}}=40 \mathrm{nLmin}^{-1}(\mathrm{\circ})$ and $X=1.9$ for $\mathrm{Q}_{\mathrm{t}}=100 \mathrm{nLmin}^{-1}(\Delta)$ determined in the present study. Comparison with the data obtained by Smith et al. ${ }^{36}$ for a similar molecular complex in shear flow using $60 \mathrm{cP}(\circ)$ and $220 \mathrm{cP}(\square)$ viscous solutions as medium and respective data fit to guide the eye, represented by the black line. The dashed region represents the asymptotic range for mean fractional extension for this similar molecular complex.

For both cases, the maximum fraction length tends to plateau at high Wi approaching values of $\sim 0.4-0.5$, corresponding to the maximum mean fractional extension for this molecule in a simple shear flow. ${ }^{36,33}$ Teixeira et $a{ }^{33}$ also found an identical plateau for the extension of a $\lambda$-DNA molecule of different contour length (of $80 \mu \mathrm{m})$, in a shear flow for a larger range of Wi numbers, reaching 600.

It is worth highlighting that, by performing a single experiment in an appropriate microfluidic customised network, we are able to characterise the full curve of mean fractional length for the whole range of relevant Weissenberg numbers (see data shown as triangles corresponding to $X=1.9, Q_{\mathrm{t}}=100 \mathrm{nLmin}^{-1}$ in Figure 6).

\section{CONCLUSIONS}

The experimental results presented in this study confirm the potential of the microfluidic bifurcating networks of customised dimensions ${ }^{21}$ to investigate the behaviour of shear sensitive molecules. The ability to customise the design of microfluidic devices a priori, according to the desired average wall shear stress distribution required for a specific application, is a major advantage.

$\lambda$-DNA is a perfect example of a shear sensitive polymer to be used for testing complex microfluidic geometries since it has been extensively studied in the past. Using the microfluidic networks, we were able to confirm the expected effect of flow strength on the molecule deformation. As predicted, different dynamics were observed for $\lambda$-DNA molecules in the two geometries tested: for $X=1$, with uniform average wall shear stress distribution, a similar moderate extension of the molecules was observed in all channels of this network; for the geometry designed with $X=1.9$ at the 
same total flow rate, the molecular deformation is stronger in the consecutive channels of the network due to the increasing shear stress imposed $(X>1)$. The data obtained on molecule deformation is in good agreement with previous studies done using $\lambda$-DNA subject to a simple shear flow between two parallel plates ${ }^{33,36}$. One of the advantages of the microfluidic networks proposed here is that by using one simple geometry and a single flow condition, we were able to cover a large range of Weissenberg numbers and assess the corresponding effect on molecule deformation.

We believe these networks have the potential to be employed in the future also for other complex fluids and are particularly valuable for cumulative shear stress studies, where the shear loading history and residence time play an important role.

\section{SUPPLEMENTARY MATERIAL}

See supplementary material for details on $\lambda$-DNA solution preparation (Section S1), discussion on the experimental limitations (Section S2) and rheological characterisation of xanthan gum solutions and respective flow kinematics inside the microfluidic channels (Section S3).

\section{ACKNOWLEDGEMENTS}

We thank Yanan Liu for precious assistance with the PTV analysis and Olivia du Roure for helpful discussions on the visualisation of $\lambda$-DNA molecules. JF, MSNO and AL thank COST Action MP1305 for financial support; LC thanks Labex-SEAM and Mairie de Paris for funding; AL acknowledges funding from the ERC Consolidator Grant PaDyFlow under Grant Agreement No. 682367; MO and KZ thank EPSRC under the auspices of Grant EP/L013118/1.

Chin, C. D., Chin, S. Y., Laksanasopin, T. \& Sia, S. K. in Point-of-Care Diagnostics on a Chip 3-21 (2013).

2. $\quad$ Kiilerich-Pedersen, K. \& Rozlosnik, Diagnostics 2, 83 (2012).

3. Li, X., Valadez, A. V., Zuo, P. \& Nie, Z. 100, 130 (2012).

4. Sharma, S., Zapatero-Rodríguez, J., Estrela, P. \& O’Kennedy, R. Biosensors 5, 577 (2015).

5. Weaver, W., Kittur, H., Dhara, M. \& Carlo, D. Di. Lab Chip 14, 1962 (2014).

6. Fidalgo, J., Dias, A., Mendes, A. M. \& Magalhaes, F. D. Chem. Eng. J. 233, 323 (2013).

7. Yang, S. et al. Lab Chip 12, 2097 (2012).

8. Lenshof, A. \& Laurell, T. Chem. Soc. Rev. 39, 1203 (2010).

9. $\quad$ Sajeesh, P. \& Sen, A. K. Microfluid. Nanofluidics 17, 1 (2014).

10. Galindo-Rosales, F. J., Alves, M. A. \& Oliveira, M. S. N. Microfluid. Nanofluidics 14, 1 (2013).

11. Pipe, C. J. \& McKinley, G. H. Mech. Res. Commun. 36, 110 (2009).

12. Lindner, A. \& Arratia, P. E. Biomicrofluidics 10, 43301 (2016).

13. Dertinger, S. K. W., Chiu, D. T., Noo Li Jeon \& Whitesides, G. M. in Anal. Chem. 73, 1240 (2001).

14. Chang, C.-W. et al. in Lab Chip 14, 3762 (2014).

15. Ishikawa, T. et al. in Biomed. Microdevices 13, 159 (2011).

16. Lu, H. et al. in Anal. Chem. 76, 5257 (2004).

17. Borenstein, J. T. et al. in Biomed. Microdevices 12, 71 (2010).

18. Huber, B. et al. in J. Funct. Biomater. 7, 11 (2016).

19. Lifton, V. A. in Lab Chip 16, 1777 (2016).

20. Zhang, Y., Park, S., Yang, S. \& Wang, T. H. in Biomed. Microdevices 12, 1043 (2010).

21. Zografos, K., Barber, R. W., Emerson, D. R. \& Oliveira, M. S. N. Microfluid. Nanofluidics 19, 737 (2015).

22. Murray, C. D. Proc Natl Acad Sci USA 207-14 (1926).

23. Alves, M. A., Oliveira, P. J. \& Pinho, F. T. Int. J. Numer. Methods Fluids 41, 47 (2003).

24. Dhinakaran, S., Oliveira, M. S. N., Pinho, F. T. \& Alves, M. A. J. Nonnewton. Fluid Mech. 198, 48 (2013).

25. Mai, D. J., Brockman, C. \& Schroeder, C. M. Soft Matter 8, 10560 (2012). 
Rems, L., Kawale, D., James Lee, L. \& Boukany, P. E. Biomicrofluidics 10, (2016).

27. Emerson, D. R., Cieślicki, K., Gu, X. \& Barber, R. W. Lab Chip 6, 447 (2006).

28. Ladoux, B., Quivy, J.-P., Doyle, P. S., Almouzni, G. \& Viovy, J.-L. Sci. Prog. 84, 267 (2001).

29. Teclemariam, N. P., Beck, V. A., Shaqfeh, E. S. G. \& Muller, S. J. Macromolecules 40, 3848 (2007).

30. Hur, J. S., Shaqfeh, E. S. G., Babcock, H. P., Smith, D. E. \& Chu, S. J. Rheol. 45, 421 (2001).

31. Bruus, H. Theoretical microfluidics. (2006).

32. Kurita, H. et al. IEEE, 295 (2010).

33. Teixeira, R. E., Babcock, H. P., Shaqfeh, E. S. G. \& Chu, S. Macromolecules 38, 581 (2005).

34. Smith, D. E., Perkins, T. T. \& Chu, S. Macromolecules 29, 1372 (1996).

35. Zeiss Support. (2016).

36. Smith, D. E., Babcock, H. P. \& Chu, S. Science 283, 1724 (1999).

37. Teixeira, R. E., Dambal, A. K., Richter, D. H., Shaqfeh, E. S. G. \& Chu, S. Macromolecules 40, 2461 (2007).

38. Schroeder, C. M., Teixeira, R. E., Shaqfeh, E. S. G. \& Chu, S. Phys. Rev. Lett. 95, 1 (2005).

39. Perkins, T. T. Science 276, 2016 (1997). 
a)

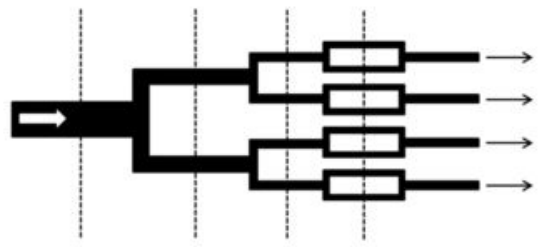

generation 0

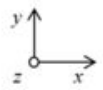

b)

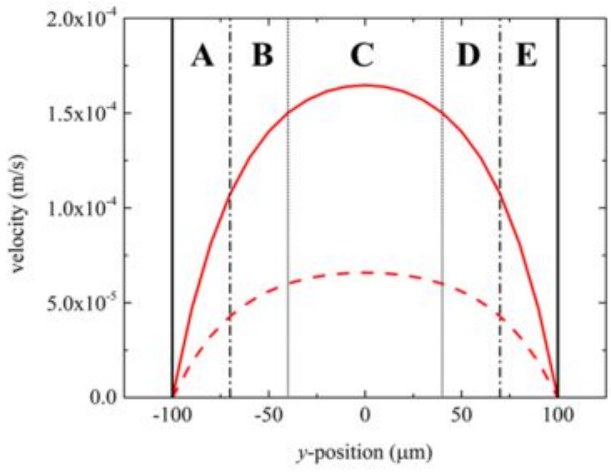



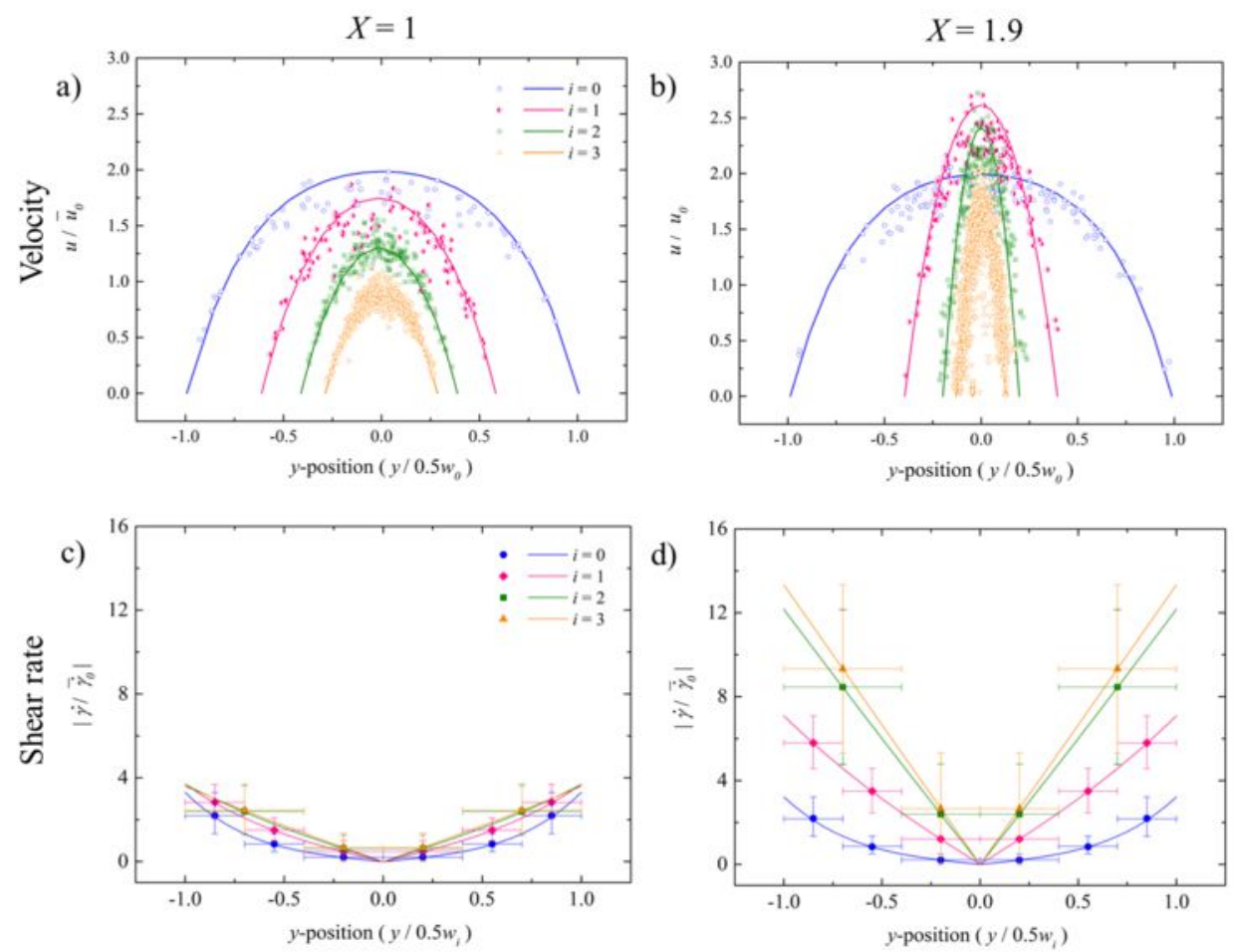
b)

$\Delta \mathrm{t}=0 \mathrm{~ms}$

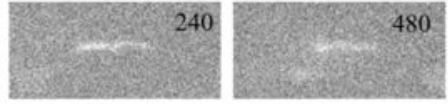

720
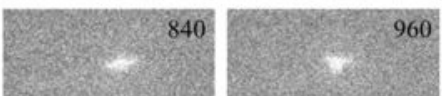

Active

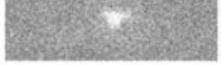

1080

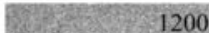

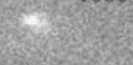

1680

1920

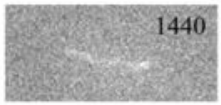

2160

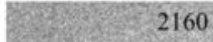

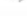

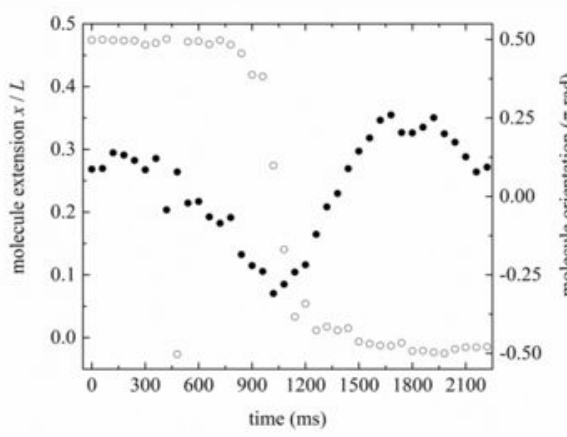

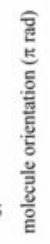




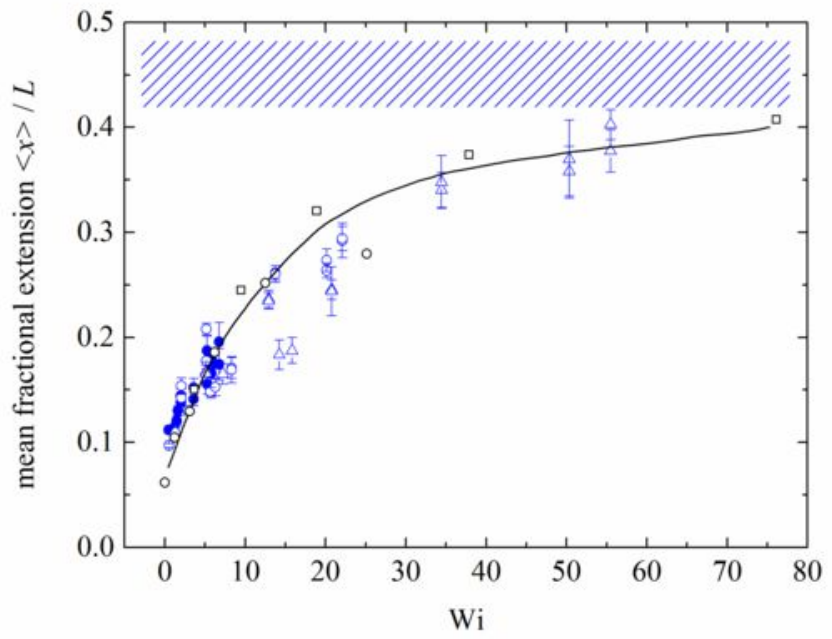


a)

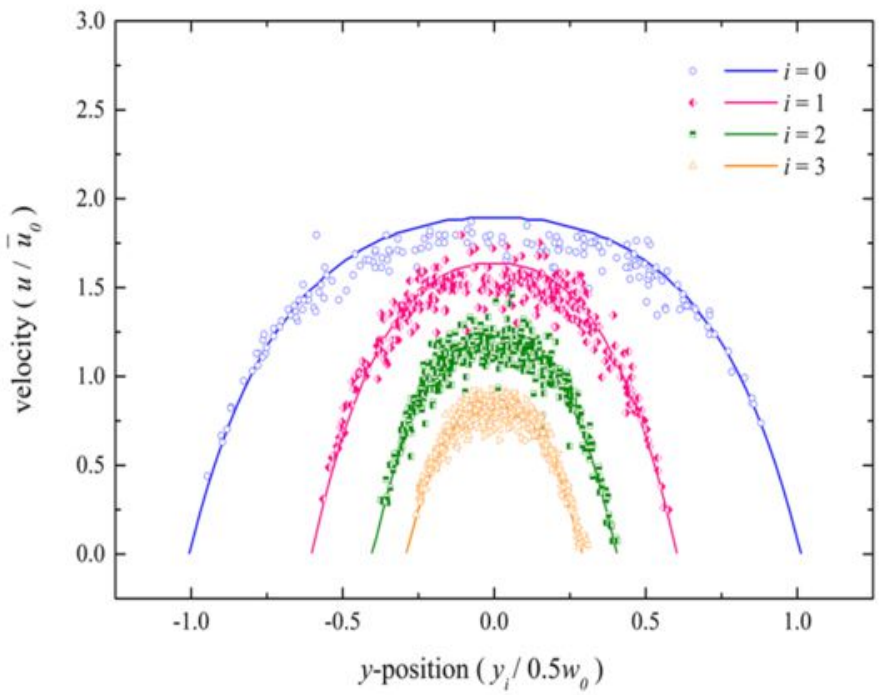

b)

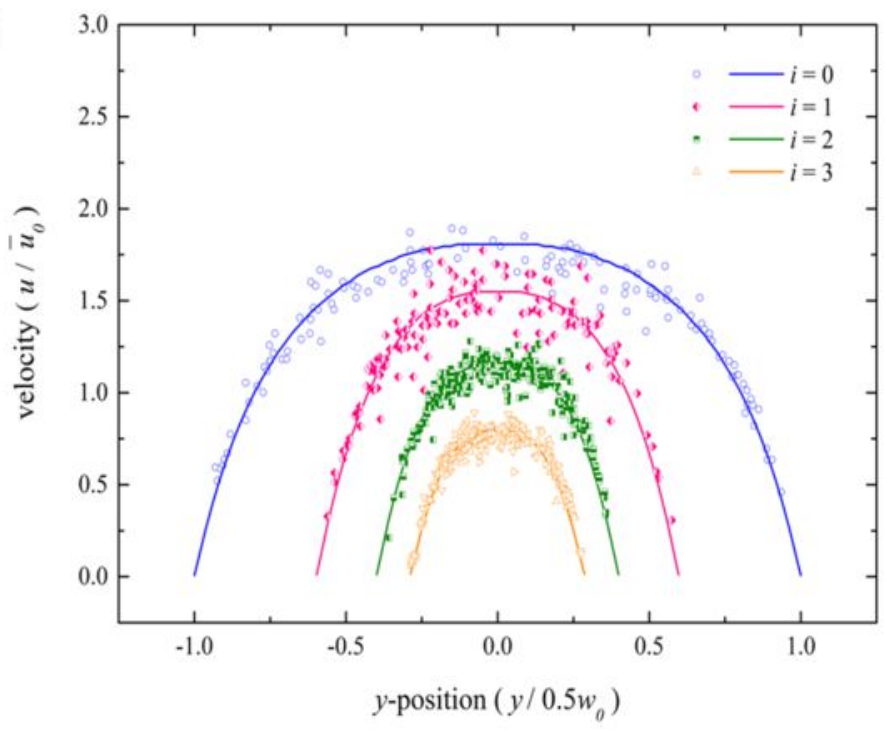




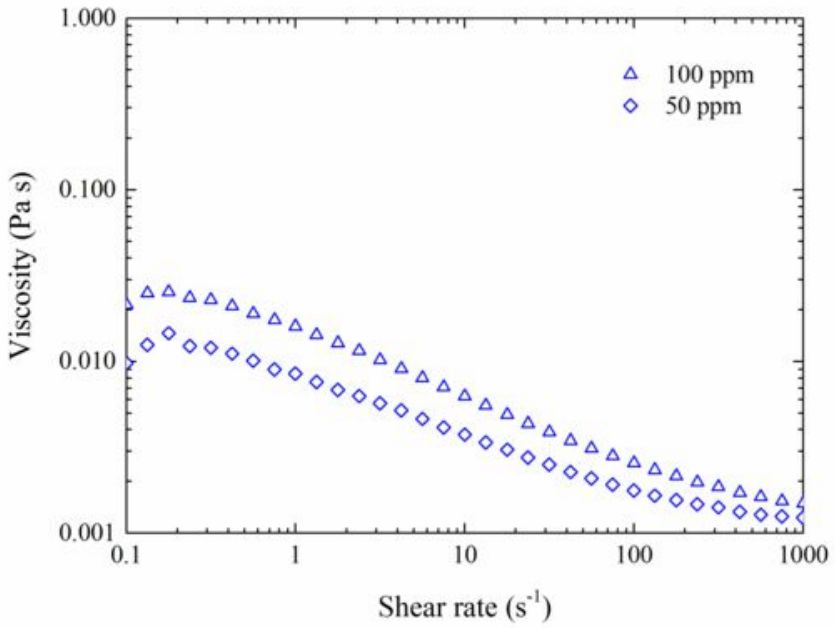




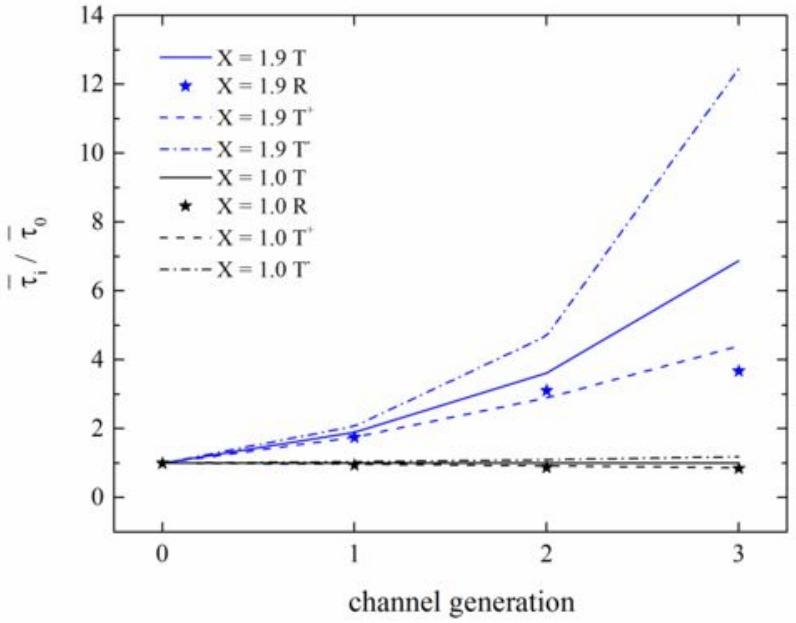



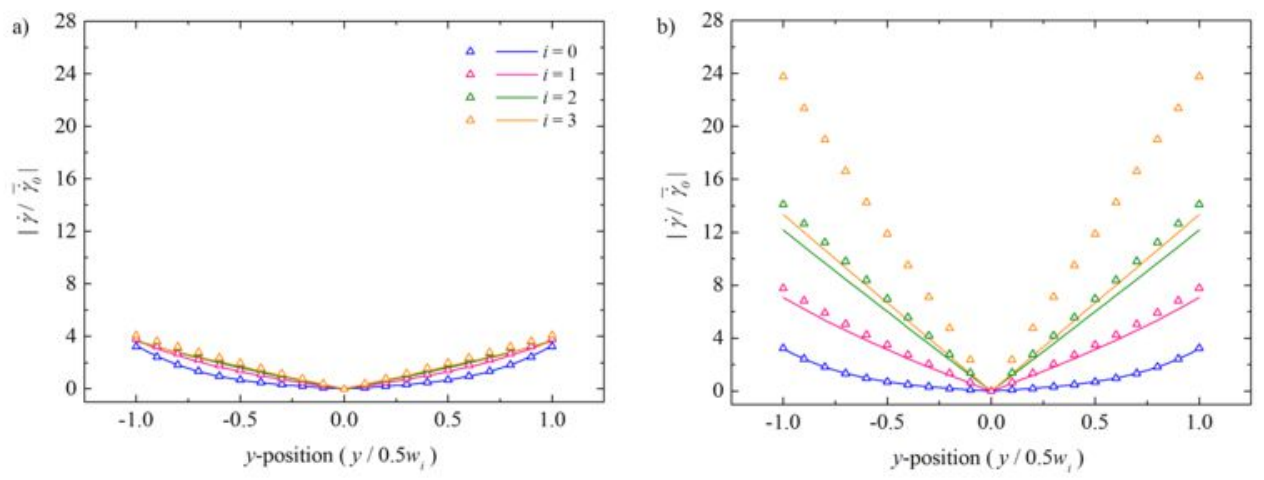